\title{
Three Dimensional Microstructural Characterization of Cathode Degradation in SOFCs Using FIB/SEM and TEM
}

\author{
Joshua Taillon $^{1}$, Christopher Pellegrinelli ${ }^{1}$, Yilin Huang ${ }^{1}$, Eric Wachsman ${ }^{1}$, and \\ Lourdes Salamanca-Riba ${ }^{1}$ \\ ${ }^{1 .}$ University of Maryland, Materials Science and Engineering, College Park, MD, USA
}

Solid oxide fuel cells (SOFC) present an efficient, clean, and flexible means of energy conversion, but the limited durability of the cells in practical applications has impeded their commercial adoption. Degradation occurs within the cathode upon long-term operation and exposure to various environmental contaminants, including $\mathrm{H}_{2} \mathrm{O}$. The contaminants cause significant microstructural and compositional changes within the cathode that adversely affect activation, polarization mechanisms, and ionic and electronic conductivities. Previous works have demonstrated that a number of quantifiable microstructural characteristics can be directly related to SOFC performance, the most important of these being triple phase boundary length $\left(\mathrm{L}_{\mathrm{TPB}}\right)$ and pore surface area [1-2]. These parameters have not been examined during cell degradation, and further analysis under these conditions provides insight into specific cell degradation mechanisms, informing future fabrication and operation criteria.

Three-dimensional quantifications of porous SOFC cathodes have been obtained after aging in both clean and contaminated atmospheres, under cathodic, anodic, and no polarization. The cathodes consisted of $50 \mathrm{wt} \% \mathrm{La}_{1-\mathrm{x}} \mathrm{Sr}_{\mathrm{x}} \mathrm{MnO}_{3}$ (LSM) and $50 \mathrm{wt} \%\left(\mathrm{Y}_{2} \mathrm{O}_{3}\right)_{0.08}-\left(\mathrm{ZrO}_{2}\right)_{0.92}$ (8-YSZ), screen printed onto a YSZ electrolyte to form LSM-YSZ /YSZ/LSM-YSZ symmetric cells. The structure of these cathodes was quantitatively analyzed using a serial nanotomography technique within the FIB/SEM (Figure 1). The reconstructed volumes were quantified by a number of parameters, including $\mathrm{L}_{\mathrm{TPB}}$, particle/pore size and distribution, surface area coverage, porosity, volume fraction, and tortuosity. Information relating to phase connectivity was compared for each cell through a skeletonization process. These parameters were analyzed in relation to electrochemical cell performance results, and correlated with composition changes observed via TEM/EELS.

Figure 1 shows four reconstructed LSM/YSZ cathodes for different aging/polarization conditions. The average reconstructed volume was approximately $4000 \mu^{3}$. After reconstruction, the ratios of segmented LSM and YSZ matched those expected from the starting source material, indicating no significant redistribution of phases occurred during aging. Analysis of the phase surface area and volume revealed that the LSM particles were significantly larger and had much lower specific surface areas (per volume) than the other phases. There did not appear to be a significant effect on these parameters from aging in either environment. Likewise, the phase distribution was anisotropic with respect to the electrolyte/cathode interface, meaning there was no significant deviation from the average, regardless of location within the cathode.

The connectivity of the phases was analyzed by computing a skeletonized representation of the segmented image data. In all LSM/YSZ cathodes, the YSZ and pore networks were found to be completely percolated, while the LSM phase was greatly fragmented, indicating that electronic conduction out of the cathode is limited in these cells. The tortuosity $(\tau)$ of each phase was also calculated in each direction by tracking the phases' two-dimensional center of mass (slice-by-slice). The ratio of phase fraction $\left(V_{p}\right)$ to tortuosity is related to the effective diffusivity $\left(D_{e f f}\right)$ in a material (and thus 
related to the conductivity) [3]. For the YSZ phase, this fraction was found to be smallest in the cells aged under $\mathrm{H}_{2} \mathrm{O}$ contamination, suggesting that the presence of water decreases $D_{\text {eff }}$ in these cells. No significant difference was observed for LSM and the pore phases.

The final microstructural property analyzed was a calculation of the triple phase boundary network. The points where three phases join represent possible sites for the oxygen reduction reaction (ORR), and provide a pathway for the reaction products to be conducted. The connectivity of the TPB network was analyzed to predict whether a portion of the network is expected to be active by determining if it is connected across the volume of the cathode that has been sampled. Isolated TPB segments were predicted to be inactive. The results of this analysis are plotted in Figure 2a-b; the results show that while both samples aged in dry air had similar active TPB fractions, the $\mathrm{H}_{2} \mathrm{O}$-Cathodic sample was significantly lower, potentially impacting performance. Finally, detailed interfacial analysis of these cathodes was performed with TEM-EELS, with which significant cation (Mn) segregation was observed (Figure 2c-d). These various parameters were then related to observed electrochemical performance. [4]

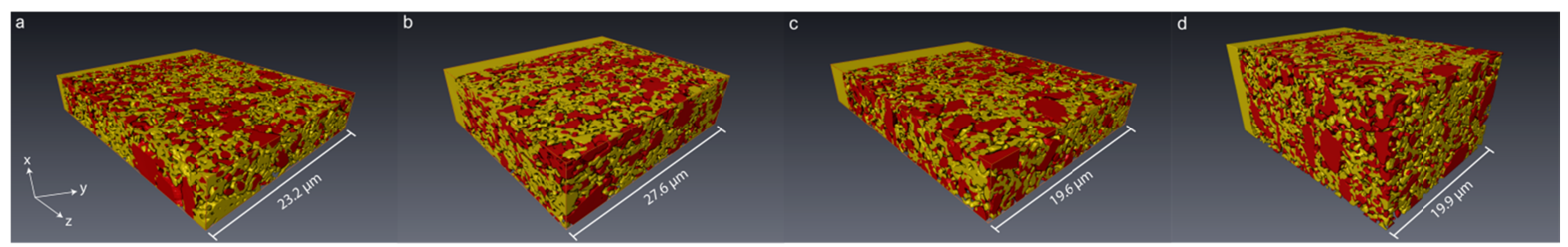

Figure 1: Visualizations of the four reconstructed LSM/YSZ composite cathodes. The aging/polarization conditions for each are (a) Air, (b) Air-Cathodic, (c) $\mathrm{H}_{2} \mathrm{O}$-Anodic, and (d) $\mathrm{H}_{2} \mathrm{O}$ Cathodic. Phases are distinguished by color: LSM in red (dark), YSZ in yellow (light), and pore phase excluded for clarity. A portion of the bulk YSZ electrolyte is visible in the rear of each reconstruction.
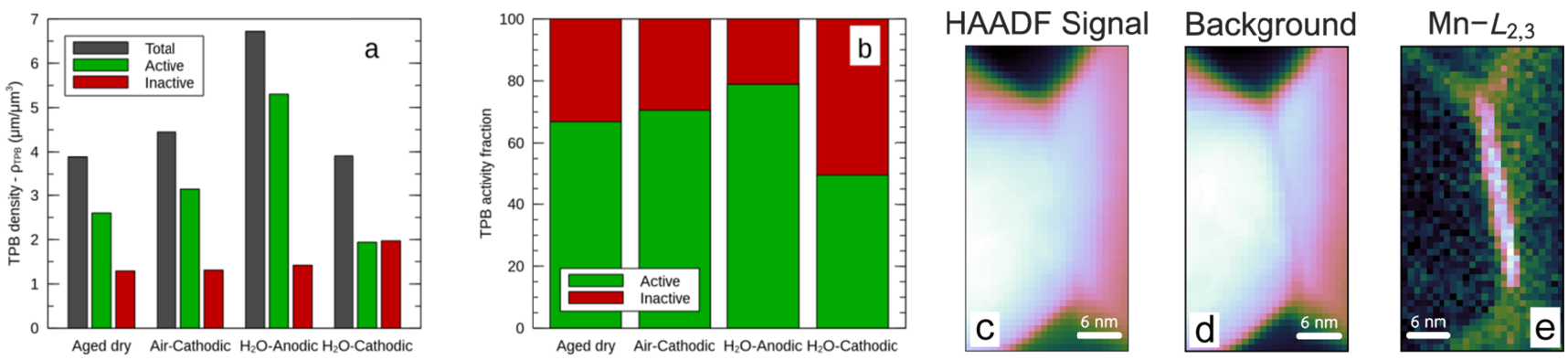

Figure 2: (a) Total, active, and inactive TPB densities for each reconstructed LSM/YSZ sample. (b) Same data as (a), comparing active vs. inactive fractions of the TPB network. (c) HAADF-STEM signal and EELS intensity maps of the background (d) and Mn- $L_{2,3}(\mathrm{e})$ edge between YSZ grains after NMF decomposition.

[1] D Gostovic et al, Journal of the American Ceramic Society 94 (2011) p. 620.

[2] JR Smith et al, Solid State Ionics 180 (2009) p. 90.

[3] CJ Gommes et al, AIChE Journal 55 (2009) p. 2000.

[4] The authors gratefully acknowledge funding from the U.S. DOE, SECA contract DEF SEE0009084. JAT additionally acknowledges funding through the NSF GRFP, grant DGE 1322106. 